\title{
43 Assessment of hearing thresholds in young children
}

A Distraction tests can be performed below 6 months of age.

B Conditioned techniques can be useful from 2 to 4 years.

C Reliable pure tone audiometry can usually be performed at a mental age of 4 years.

D A hearing aid can be used to assess hearing ability.

E Blinking, frowning and stilling are responses to sound of a normal 3 month child.

\section{Electronic hearing aids}

A In NHS aids the letter ' $L$ ' denotes a high frequency emphasis with a low frequency cut-off.

B A low frequency cut may be very useful in severe high frequency losses.

C Vented earmoulds are useful for 'ski slope' type audiograms.

D Peak clipping in sensorineural hearing loss with a reduced dynamic range may lead to distortion.

$E$ The switch marked ' $T$ ' is for use in the presence of an inductive loop system. 